Publ. RIMS, Kyoto Univ.

13 (1977), 681-686

\title{
Modifications Continues des Variétés de Stein
}

\author{
Par \\ Tetsuo UEDA*
}

\section{\$1. Modifications Continues de Dimension 2}

Une modification continue est définie par un quintuplet $T=(\widetilde{M}, S$, $\tau, N, M)$ satisfaisant aux conditions suivantes:

(1) $\widetilde{M}$ et $M$ sont des variétés analytiques complexes.

(2) $S$ est un ensemble analytique de codimension 1 dans $\widetilde{M}$.

(3) $N$ est un ensemble mince dans $M$.

(4) $\tau$ est une application holomorphe de $\widetilde{M}$ dans $M$, telle que sa restriction à $\widetilde{M} \backslash S$ soit un homéomorphisme de $\widetilde{M} \backslash S$ sur $M \backslash N$. (Voir [1])

Le présent mémoire a pour but de chercher, dans le cas de dimension 2 , des conditions pour que $\widetilde{M}$ soit une variété de Stein.

Si $\widetilde{M}$ est une variété de Stein, $N$ est un ensemble analytique de codimension 1 dans $M$. En effet, comme $\widetilde{M}$ est une variété de Stein, $\widetilde{M} \backslash S \cong M \backslash N$ le sont aussi. $M \backslash N$ est alors pseudoconvexe dans $M$. Or $N$ est mince par définition. D’après le théorème de continuité, $N$ est un ensemble analytique de codimension 1.

On peut supposer, sans restreindre la généralité, que $\widetilde{M} \backslash S$ soit le domaine maximum dans lequel $\tau$ est injective. Une telle modification est dite essentielle. Dans tout ce qui suit, nous supposerons que $T$ est une modification continue essentielle de dimension 2: $\operatorname{dim} \widetilde{M}=\operatorname{dim} M=2$.

$T$ est dite de type fini, si $S$ consiste en un nombre fini de composantes irréductibles. $T$ est appelée $\sigma$-modification, si $\widetilde{M}$ est obtenue à partir de $M$ par une itération de $\sigma$-processus en nombre fini de fois. Une $\sigma$-modification est de type fini. Une modification de type fini est considérée comme une restriction d'une $\sigma$-modification d'après le théorème suivant:

Communiqué par S. Nakano, novembre le 9, 1976.

* Département de Mathématique, Université de Kyoto, Kyoto 606, Japon. 
Théorème. (Stoll, [3] Satz 4.1). Soit T une modification de type fini. Alors il existe une $\sigma$-modification $\Lambda=\left(M^{*}, S^{*}, \lambda, \tau(S), M\right)$ et une application holomorphe injective $\iota: \widetilde{M} \rightarrow M^{*}$ telle que $\lambda \circ \mathrm{c}=\tau$.

En ce moment, si l'on suppose que $\widetilde{M}$ soit une variété de Stein, $N^{*}=M^{*} \backslash \iota(\widetilde{M})$ est un ensemble analytique de codimension 1 dans $M^{*}$ d'après le théorème de continuité. Comme on a $S \cong \lambda^{-1}(N) \backslash N^{*}$, toute composante de $S$ est analytiquement homéomorphe à la partie de la sphère de Riemann obtenue par enlèvement d'un nombre fini de points.

Étant donnée une modification, on peut former une modification de type fini, en détachant de $\widetilde{M}$ presque toutes les composantes irréductibles de $S$ sauf un nombre fini d'elles. Ce détachement ne change pas la propriété de Stein. On en conclut l'enoncé suivant: Si $\widetilde{M}$ est une variété de Stein, toute composante irréductible de $S$ est analytiquement homéomorphe a une courbe rationnelle pointée en un nombre fini de points.

\section{§ 2. Modifications Continues de Dimension 2 d'Ordre 1}

Nous envisageons, dans cette section, le cas où toute composante irréductible $S_{j}$ de $S$ est obtenue par un $\sigma$-processus au point $\tau\left(S_{j}\right)$ de $M$. Nous appelons une telle modification d'ordre 1 .

Théorème 1. Soit $T=(\widetilde{M}, S, \tau, N, M)$ une modification continue essentielle de dimension 2 d'ordre 1. Supposons que les conditions suivantes sont remplies:

i) $M$ est une variété de Stein.

ii) $N$ est un ensemble analytique de codimension 1 dans $M$.

iii) Toute $S_{j}$ est analytiquement homéomorphe à $\mathbb{C}=\mathbb{P}^{1} \backslash\{\infty\}$.

iv) L'ensemble $\tau(S)$ est situé sur la portion régulière de $N$.

v) L'ensemble $\tau(S)$ est discret dans $N$.

Alors $\widetilde{M}$ est une variété de Stein.

Pour montrer le théorème, commençons par construire une fonction méromorphe $f$ dans $M$ qui admet ses poles d'ordre 1 justement sur $N$, 
et s'exprime en tout point $\tau\left(S_{j}\right)$ sous la forme $f=\frac{y}{x}$ par rapport à un système de coordonnées locales $(x, y)$ convenable tel que $N=\{x=0\}$ et $\tau\left(S_{j}\right)=(0,0)$.

Soit $\left\{\left(\phi_{i}, U_{i}\right)\right\}$ une donnée du deuxième problème de Cousin qui définit $N: \phi_{i}$ s'annulent avec l'ordre 1 sur $N \cap U_{i},\left\{U_{i}\right\}$ étant un recouvrement ouvert de $M$. Posons $u_{i j}=\phi_{i} / \phi_{j}$ dans $U_{i} \cap U_{j}$. Pour le cocycle $\left\{\left(u_{i j}, U_{i} \cap U_{j} \cap N\right)\right\} \in Z^{1}\left(N, \mathcal{O}^{*}\right)$, on peut trouver $\left\{\left(a_{i}, U_{i} \cap N\right)\right\} \in C^{0}(N$, $\left.\mathcal{O}^{*}\right)$ tel que $a_{i} / a_{j}=u_{i j}$ dans $U_{i} \cap U_{j} \cap N$, puisque $H^{1}\left(N, \mathcal{O}^{*}\right)=0$. En multipliant $a_{i}$ par une fonction holomorphe sur $N$ convenable, on peut former un système $\left\{\left(b_{i}, U_{i} \cap N\right)\right\}$ tel que chaque $b_{i}$ admette comme zéros d'ordre 1 tous les points de $\tau(S)$ dans $U_{i} \cap N$. En prolongeant $b_{i}$ en une fonction holomorphe $\tilde{b}_{i}$ dans $U_{i}$, on a un système $\left\{\left(\tilde{b}_{i}, U_{i}\right)\right\}$, tel qu'on ait $\left.\tilde{b}_{i}\right|_{U_{i}} \cap_{N}=b_{i}$ et $\tilde{b}_{i} / \tilde{b}_{j}=u u_{i j}=\phi_{i} / \phi_{j}$ sur $U_{i} \cap U_{j} \cap N . \quad\left\{\left(\tilde{b}_{i} / \phi_{i}, U_{i}\right)\right\}$ est une donnée du premier problème de Cousin, dont la résolution $f$ est manifestement une fonction voulue.

Considérons l'adhérence $G$ dans $M \times \mathbb{C}$ de l'ensemble

$$
\{(p, f(p)) ; p \in M \Lambda N\}
$$

$G$ est une sous-variété régulièrement plongée dans $M \times \mathbb{C}$, et par conséquent une variété de Stein. On a, d'une façon canonique, une application holomorphe injective $\tau_{1}: \widetilde{M} \rightarrow G$ où $G \backslash \tau_{1}(\widetilde{M})$ est vide ou un ensemble analytique de codimension 1 dans $G$. $\widetilde{M}$ est donc une variété de Stein. c.q.f.d.

Theoremè 2. Soit $T$ une modification continue de dimension 2. S'il existe une suile de composantes irréductibles $\left\{S_{\nu}\right\}_{\nu}$ de $S$, telle que i) toute $S_{\nu}$ soit d'ordre 1 , ii) les $\tau\left(S_{\nu}\right)$ tendent vers un point régulier $p$ de $N$, alors $\widetilde{M}$ ne peut être une variété de Stein.

Soit $U$ un voisinage de $p$ dans $M$ avec un système de coordonnées locales $(x, y): U=\{|x|<1,|y|<1\}, N \cap U=\{x=0,|y|<1\} . \quad(x(p), y(p))$ $=(0,0),\left(x\left(\tau\left(S_{\nu}\right)\right), y\left(\tau\left(S_{\nu}\right)\right)\right)=\left(0, y_{\nu}\right)$.

Soit $K$ un compact dans $\widetilde{M}$ de la forme: $K=\left\{q \in \tau^{-1}(U): r^{\prime} \leqq\right.$ $|x(\tau(q))| \leqq r,|y(\tau(q))| \leqq r\}, r$ et $r^{\prime}$ étant des nombres réels tels que $0<r^{\prime}$ $<r<1$. On va montrer que l'enveloppe $\widehat{K}$ de $K$ par rapport aux fonc- 
tions holomorphes dans $\widetilde{M}: \widehat{K}=\left\{q \in M:|f(q)| \leqq \max _{q^{\prime} \in K}\left|f\left(q^{\prime}\right)\right|\right.$ pour toute $f$ holomorphe dans $\widetilde{M}\}$, n'est pas compacte, ce qui prouvera que $\widetilde{M}$ n'est pas holomorphiquement convexe.

Considérons, pour chaque $\nu$, l'ensemble analytique dans $\tau^{-1}(U)$ donné par $\left\{q \in \tau^{-1}(U): y(\tau(q))=y_{\nu}\right\}$, qui se compose de deux composantes irréductibles, dont l'une est $S_{\nu}$. Nous notons l'autre $L_{\nu}$. $L_{\nu}$ est homéomorphe par la fonction $x \circ \tau$ à un disque $\{|x|<1\}$ dans le plan complexe. $L_{\nu}$ et $S_{\nu}$ se recontrent en un point $q_{\nu}$. Or, pour tout $\nu$ tel que $\left|y\left(\tau\left(q_{\nu}\right)\right)\right|$ $\leqq r, q_{\nu}$ est entouré dans $L_{\nu}$ par l'ensemble $K \cap L_{\nu}$. Par le principe du maximum, $q_{\nu}$ est compris dans $\hat{K}$. $\hat{K}$ n'est pas alors compacte, puisqu'elle comprend les points $q_{\nu}$ qui forment un ensemble discret dans $\widetilde{M}$. c.q.f.d.

Soit $T$ une modification continue essentielle de dimension 2 d'ordre 1 qui remplit les conditions i) $\sim$ iv) du théorème 1 . Pour que $\widetilde{M}$ soit une variété de Stein, il faut que $\tau(S)$ ne s'accumule à aucun point régulier de $N$, d'après le théorème 2. Qu'arrivera-t-il dans le cas où $\tau(S)$ s'accumule seulement à des points singuliers de $N$ ? L'auteur n'arrive pas encore à une solution générale. Nous indiquons que la condition est assez délicate, en en donnant deux exemples: pour l'un $\widetilde{M}$ est une variété de Stein et pour l'autre elle ne l'est pas.

Exemples. Soient $\Delta$ un dicylindre $\{|x|<1,|y|<1\}$ dans l'espace $\mathbb{C}^{2}(x, y)$ et $N=\{x y=0\} \cap \Delta . \quad$ Soit $T=(\tilde{\Delta}, S, \tau, N, \Delta)$ une modification continue essentielle d'ordre 1 qui remplit les conditions i) iv) du théorème 1 et en outre la condition suivante:

$\left.\mathrm{v}^{\prime}\right) \quad \tau(S)$ est discret dans la portion régulière de $N$.

Soient $S_{j}{ }^{\prime}$ [resp. $\left.S_{j}{ }^{\prime \prime}\right]$ les composantes irréductibles de $S$ telles que $\tau\left(S_{j}{ }^{\prime}\right)$ $=\left(x_{j}, 0\right) \quad\left[\operatorname{resp} .\left(S_{j}^{\prime \prime}\right)=\left(0, y_{j}\right)\right],(j=1,2, \cdots)$.

Si un au moins des ensembles $\left\{\tau\left(S_{j}{ }^{\prime}\right)\right\}$ et $\left\{\tau\left(S_{j}{ }^{\prime \prime}\right)\right\}$ ne s'accumule pas à $(0,0), \tilde{J}$ est une variété de Stein.

En effet, supposons que $\left\{\tau\left(S_{j}^{\prime}\right)\right\}$ ne s'accumule pas à $(0,0)$. Soit $\Delta^{*}$ une variété complexe obtenue à partir de $\Delta$ par un $\sigma$-processus à chaque point de $\left\{\tau\left(S_{j}^{\prime}\right)\right\}$. Soient $\lambda: \Delta^{*} \rightarrow \Delta$ et $\mu: \tilde{\Delta} \rightarrow \Delta^{*}$ les applications définies d'une façon naturelle. Posons $\Delta^{\prime}=\Delta^{*} \backslash \overline{\lambda^{-1}\left(\{y=0\} \backslash\left\{\tau\left(S_{j}^{\prime}\right)\right\}\right)} .\left.\quad \lambda\right|_{\Delta^{\prime}}: \Delta^{\prime} \rightarrow \Delta$ engendre une modification qui satisfait aux conditions du théorème $1 . \Delta^{\prime}$ 
est donc une variété de Stein. $\mu: \tilde{\Delta} \rightarrow \Delta^{\prime}\left(\mu(\tilde{\Delta}) \subseteq \Delta^{\prime}\right)$ engendre aussi une modification à laquelle s'applique le théorème 1 . D'où vient l'énoncé.

Le cas qui nous intéresse est celui où $\left\{\tau\left(S_{j}{ }^{\prime}\right)\right\}$ et $\left\{\tau\left(S_{j}{ }^{\prime \prime}\right)\right\}$ s'accumulent tous deux vers $(0,0)$.

a) S'il existe des suites partielles $\left\{x_{j(\nu)}\right\}_{\nu}$ et $\left\{y_{k(\nu)}\right\}_{\nu}$ telles que $x_{j(\nu)} \rightarrow 0, y_{k(\nu)} \rightarrow 0$ et $\frac{y_{k(\nu)}}{x_{j(\nu)}} \rightarrow c$, avec $\nu \rightarrow \infty$, c étant un nombre complexe différent de zéro, alors $\tilde{J}$ n'est pas une variété de Stein.

La démonstration est pareille à celle du théorème 2 .

Prenons un ensemble dans $\tilde{\Delta}$ de la forme

$$
\{y(\tau(p))+c x(\tau(p))=0,|x(\tau(p))|=r\}, 0<r<\min \left\{1, \frac{1}{|c|}\right\},
$$

et soit $K$ son voisinage compact. On va montrer que l'enveloppe $\widehat{K}$ de $K$ par rapport aux fonctions holomorphes dans $\tilde{\Delta}$ n'est pas compacte. Soit, pour chaque $\nu, L_{\nu}$ l'ensemble analytique qui correspond par $\tau$ à la droite complexe passant par $\left(x_{j(\nu)}, 0\right)$ et $\left(0, y_{k(\nu)}\right) . \quad L_{\nu}$ rencontre $S_{j(\nu)}^{\prime}$ en un point $p_{\nu}{ }^{\prime}$ et $S_{k(\nu)}^{\prime \prime}$ en un point $p_{\nu}{ }^{\prime \prime}$. Pour $\nu$ suffisamment grand, $p_{\nu}{ }^{\prime}$ et $p_{\nu}{ }^{\prime \prime}$ sont entourés dans $L_{\nu}$ par l'ensemble $K \cap L_{\nu}$. $p_{\nu}{ }^{\prime}$ et $p_{\nu}{ }^{\prime \prime}$ sont donc compris dans $\hat{K}$, ce qui montre que $\hat{K}$ n'est pas compacte. c.q.f.d.

b) Si, pour $\left\{x_{j}\right\}$ et $\left\{y_{j}\right\}$, les suites $\left\{\frac{x_{n+1}}{y_{1} \cdots y_{n}}\right\}$ et $\left\{\frac{y_{n}}{x_{1} \cdots x_{n}}\right\}$ tendent à la fois vers zéro, $\tilde{\Delta}$ est une variété de Stein.

Pour le voir, prenons d'abord une suite de nombres positifs $\left\{r_{n}\right\}_{n=1}^{\infty}$, décroissant et tendant vers zéro, telle que $\left|\frac{y_{n}}{x_{1} \cdots x_{n}}\right|<r_{2 n-1}$ et $\left|\frac{x_{n+1}}{y_{1} \cdots y_{n}}\right|$ $<r_{2 n}$ pour tout $n$. Formons une suite de domaines $\left\{D_{n}\right\}_{n=1}^{\infty}$ dans $\tilde{\Delta}$ :

où $\quad f_{n}(p)=\frac{1}{y \circ \tau(p)} \prod_{j=1}^{n} \frac{x \circ \tau(p)-x_{j}}{1-\bar{x}_{j} x \circ \tau(p)}$,

$$
\begin{aligned}
& D_{2 n-1}=\left\{\left|f_{n}\right|<\frac{1}{r_{2 n-1}},\left|g_{n}\right|<\frac{1}{r_{2 n-1}}\right\} \\
& D_{2 n}=\left\{\left|f_{n+1}\right|<\frac{1}{r_{2 n}},\left|g_{n}\right|<\frac{1}{r_{2 n}}\right\}
\end{aligned}
$$

et

$$
g_{n}(p)=\frac{1}{x \circ \tau(p)} \prod_{j=1}^{n} \frac{y \circ \tau(p)-y_{j}}{1-\bar{y}_{j} y \circ \tau(p)}
$$


On remarque que ces fonctions $f_{n}$ et $g_{n}$ sont méromorphes et n'admettent aucun point d'indétermination dans $\tilde{\Delta}$. On voit aisément que $D_{n} \subset D_{n+1}$ et que $\cup D_{n}=\tilde{\Delta}$.

De plus, les fonctions qui definissent $D_{n}$ sont holomorphes dans $D_{n+1}$ 。 En effet, envisageons une paire $D_{2 n-1}$ et $D_{2 n}$, par exemple. $f_{n}$ admet les pôles sur $S_{j}^{\prime}(j \geqq n+1)$ et $g_{n}$ sur $S_{j}{ }^{\prime \prime}(j \geqq n+1)$. $S_{j}{ }^{\prime}(j \geqq n+2)$ et $S_{j}{ }^{\prime \prime}$ $(j \geqq n+1)$, étant les pôles de $f_{n+1}$ et $g_{n}$ respectivement, sont à l'extérieur de $D_{2 n}$. Or $g_{n}$ prend la valeur $\frac{1}{x_{n+1}}-\prod_{j=1}^{n}\left(-y_{j}\right)$ sur $S_{n+1}^{\prime}$, dont le module est $\left|\frac{y_{1} \cdots y_{n}}{x_{n+1}}\right|>\frac{1}{r_{2 n}}$ par hypothèse, ce qui montre que $S_{n_{+1}}^{\prime}$ est à l'extérieur de $D_{2 n}$. Il en est de même pour toute paire $D_{2 n}$ et $D_{2 n+1}$.

$\hat{j}$ est ainsi limite d'une suite croissante de variétés de Stein $\left\{D_{n}\right\}$. tout $D_{n}$ étant convexe par rapport aux fonctions holomorphes dans $D_{n+1}$. $\jmath$ est donc une variété de Stein.

c.q.f.d.

\section{Références}

[1] Grauert, H. und Remmert, R., Zur Theorie der Modifikationen. I. Stetige und eigentliche Modifikationen komplexer Räume, Math. Ann., 129 (1955), 274-296.

[2] Stoll, W., Über meromorphe Modifikationen. IV. Die Erzeugung analytischer und meromorpher Modifikationen zwischen kompakten Mannigfaltigkeiten durch $\sigma$-Prozesse, Math. Ann., 130 (1955), 147-182.

[3] — Über meromorphe Modifikationen. V. Die Erzeugung analytischer und meromorpher Modifikationen durch $\sigma$-Prozesse, Math. Ann., 130 (1955), 272-316. 